\title{
Amputation with Median Nerve Redirection (Targeted Reinnervation) Reactivates Forepaw Barrel Subfield in Rats
}

\author{
Paul D. Marasco ${ }^{1}$ and Todd A. Kuiken ${ }^{1,2,3}$ \\ ${ }^{1}$ Neural Engineering Center for Artificial Limbs, Rehabilitation Institute of Chicago, Chicago, Illinois 60611, ${ }^{2}$ Department of Physical Medicine and \\ Rehabilitation, Northwestern University, Chicago, Illinois 60611, and ${ }^{3}$ Department of Biomedical Engineering, Northwestern University, Evanston, Illinois \\ 60208
}

Prosthetic limbs are difficult to control and do not provide sensory feedback. Targeted reinnervation was developed as a neural-machine interface for amputees to address these issues. In targeted reinnervation, amputated nerves are redirected to proximal muscles and skin, creating nerve interfaces for prosthesis control and sensory feedback. Touching the reinnervated skin causes sensation to be projected to the missing limb. Here we use electrophysiological brain recording in the Sprague Dawley rat to investigate the changes to somatosensory cortex (S1) following amputation and nerve redirection with the intent to provide insight into the sensory phenomena observed in human targeted reinnervation amputees. Recordings revealed that redirected nerves established an expanded representation in S1, which may help to explain the projected sensations that encompass large areas of the hand in targeted reinnervation amputees. These results also provide evidence that the reinnervated target skin could serve as a line of communication from a prosthesis to cortical hand processing regions. S1 border regions were simultaneously responsive to reinnervated input and also vibrissae, lower lip, and hindfoot, suggesting competition for deactivated cortical territory. Electrically evoked potential latencies from reinnervated skin to cortex suggest direct connection of the redirected afferents to the forepaw processing region of S1. Latencies also provide evidence that the widespread reactivation of S1 cortex may arise from central anatomical interconnectivity. Targeted reinnervation offers the opportunity to examine the cortical plasticity effects when behaviorally important sensory afferents are redirected from their original location to a new skin surface on a different part of the body.

\section{Introduction}

Prosthetic devices are difficult for amputees to control due to a lack of adequate motor command pathways and an absence of sensory feedback. Targeted reinnervation is a neural-machine interface that was developed to make prosthetic control and feedback more intuitive by using physiologically appropriate channels.

Limb nerves left following an amputation are surgically redirected to new skin and muscle sites (Hijjawi et al., 2006). The redirected nerves reinnervate the denervated target muscles and skin. When controlling a prosthesis with the redirected nerves, amputees attempt to move their missing limb. The redirected nerves signal muscle contractions that generate electromyographic signals corresponding to the intended movement. These signals are used to simultaneously control multiple joints of robotic arms (Kuiken et al., 2004, 2007b; Zhou et al., 2007). When targeted reinnervation amputees are touched on the target skin, they feel as though they are being touched on their missing limb

Received Aug. 3, 2010; revised Sept. 28, 2010; accepted 0ct. 4, 2010.

This work was supported by National Institutes of Health Grants N01-HD-5-3402, R01-HD-4-3137, and R01-HD4-4798, the Searle Funds at the Chicago Community Trust, and the Defense Advanced Research Projects Agency. We thank S. Sharma, A. Schultz, G. Dumanian, J. Ko, and S. Paisley-Agnew.

Correspondence should be addressed to Dr. Paul D. Marasco at his present address: Advanced Platform Technology (APT) Center, Louis Stokes Cleveland Department of Veterans Affairs Medical Center, 10701 East Boulevard, 151 AW/APT, Cleveland, OH 44106. E-mail: pmarasco@aptcenter.org.

DOI:10.1523/JNEUROSCI.4044-10.2010

Copyright $\odot 2010$ the authors $\quad 0270-6474 / 10 / 3016008-07 \$ 15.00 / 0$
(Kuiken et al., 2007a,b). Kuiken et al. (2007a) shows the percepts to touch that arose in the target skin of two human targeted reinnervation amputees. There are many interesting outcomes related to the redirection of the sensory nerves in targeted reinnervation amputees. The amputees have a strong impression that the sensations arising from stimulation of the reinnervated skin are projected to their missing limb (Kuiken et al., 2007a). When the reinnervated skin is touched, the amputees typically feel large portions of their missing hand, and the projected sensations often appear to encompass the cutaneous distributions of the individual transferred nerves (Kuiken et al., 2007a). The projected sensation also appears to be different from the phenomenon of phantom sensation, which is likely a result of functional reorganization of central representations (Ramachandran et al., 1992a,b; Borsook et al., 1998; Flor et al., 1998, 2000). The reinnervated skin of the amputees exhibits high functionality. For example, pressure discrimination with the reinnervated target skin is at near normal levels for the hand (Kuiken et al., 2007a; Sensinger et al., 2009). Vibration detection thresholds reflect contralateral normal skin (Schultz et al., 2009), and tactile acuity is returned to the target skin, in some cases exceeding the sensory capacities of the contralateral normal skin despite a lower intrinsic target skin innervation density (Marasco et al., 2009).

With this work, we seek to better understand the cortical effects from redirecting behaviorally important afferents from an amputated forelimb to a different skin surface. Earlier studies of cortical plasticity, following large-scale alterations to sensory in- 
A

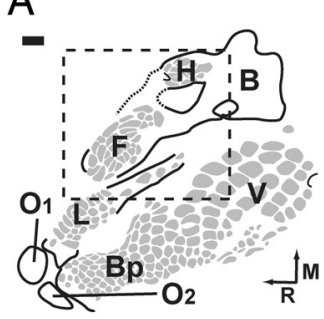

$\mathrm{F}$

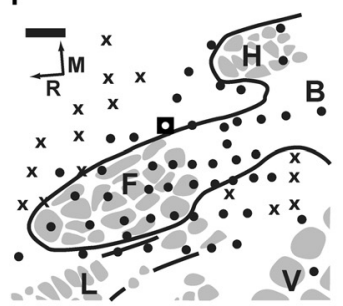

$\mathrm{K}$

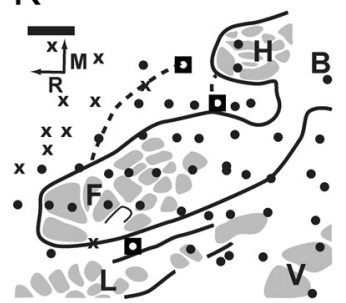

B

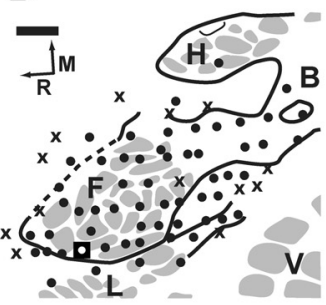

$G$

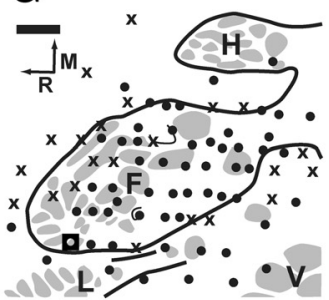

$L$

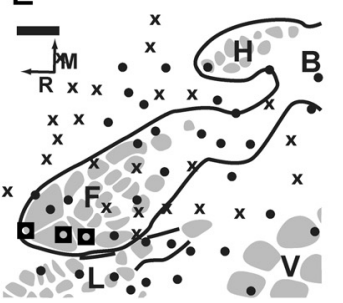

C

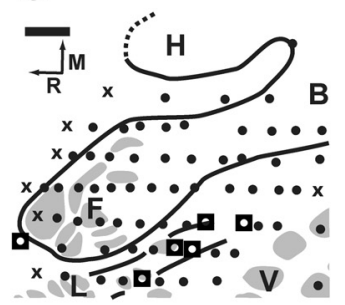

$\mathrm{H}$

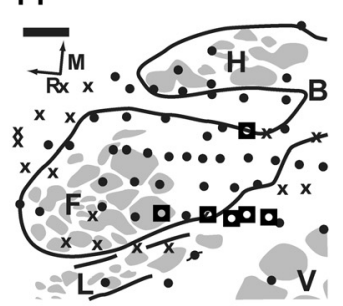

$\mathrm{M}$

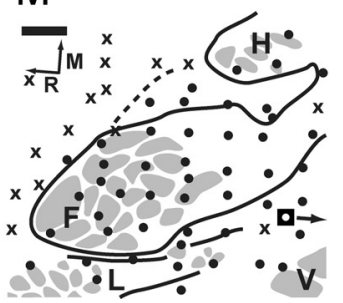

D
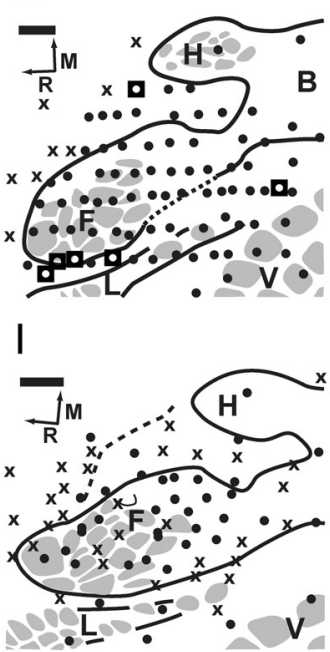

$\mathrm{N}$

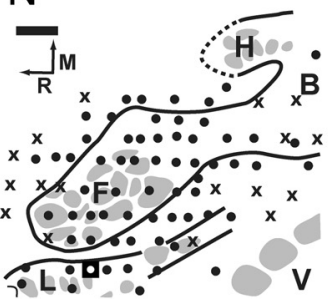

E

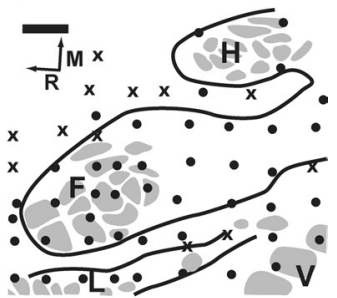

$\mathrm{J}$

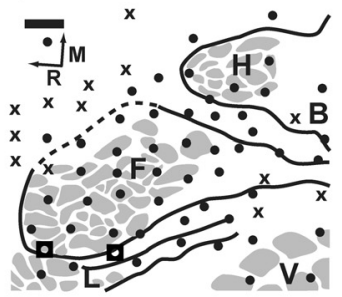

$\mathrm{O}$

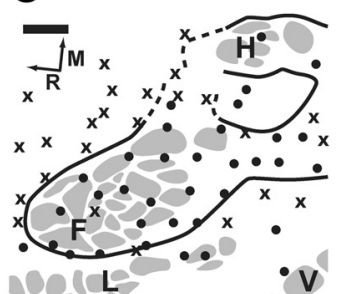

Figure 1. Results of microelectrode recordings in the $S 1$ cortex of the left hemispheres of the 14 amputated targeted reinnervation rats. $A$, Complete map of $S 1$ to reference organization. $F$, Forepaw barrel subfield; $H$, hindpaw barrel subfield; $B$, body; V, vibrissae; $C$, chin/lower lip; 01, oral module 1;02, oral module 2; L, lower lip; Bp, buccal pad; $R$, rostral; $M$, medial. Gray spots delineate barrel patterns within $\mathbf{S 1}$, and black lines define the borders of the $\mathbf{S 1}$ representation. Dashed square shows the area of the forelimb barrel subfield that is the focus of panels $\boldsymbol{B}-\boldsymbol{O}$. Barrel patterns within the forelimb barrel subfield correspond to digits of the forepaw. $\boldsymbol{B}-\mathbf{0}, \bullet$, Responsive site; $\times$, unresponsive site; $\boldsymbol{\square}$, dual receptive field site. The forelimb barrel subfield was found to be largely active in these animals, although only the median nerve was redirected to the target skin of the residual limb. In $\boldsymbol{I}$ and $\boldsymbol{L}-\mathbf{0}$, there was a region of silent cortex between the active forelimb barrel subfield and the intact body. Three of the animals did not show dual receptive fields. Scale bars, $500 \mu \mathrm{m}$.

put, have used skin island transfers, nerve repair, and limited deafferentation (Paul et al., 1972; Merzenich et al., 1983; Wall et al., 1986; Merzenich and Jenkins, 1993; Jain et al., 1997). The nerve redirection and hyper-reinnervation model described here provides a new tool to investigate mechanisms of central plasticity following cortical deactivation and reactivation (Kuiken et al., 1995, 2004). This new model also provides preliminary insight into the sensory outcomes observed in human targeted reinnervation amputees.

\section{Materials and Methods}

Cortical mapping in adult male Sprague Dawley rats. Eighteen Sprague Dawley rats were used for this study; 14 animals underwent amputation and targeted reinnervation and 4 control animals underwent amputation only. All 18 animals underwent electrophysiological recording following recovery. We used the rat as a model for these experiments because they have a well developed and well characterized cortical limb representation (Welker, 1971; Wall and Cusick, 1984; Dawson and Killackey, 1987; Waters et al., 1995) and they use their forepaws for dexterous manipulations in many ways that are similar to humans (Iwaniuk and Whishaw, 2000; Cenci et al., 2002). All procedures were approved by the Northwestern University Animal Care and Use Committee and followed National Institutes of Health guidelines.

Amputation with targeted reinnervation. Targeted reinnervation animals (average weight $=361.9 \mathrm{~g}, \mathrm{SD}=30.3$ ) were anesthetized with $2.5 \%$ isoflurane in $100 \%$ oxygen (VetEquip). Body temperature was maintained with a water-circulating heating pad. Under aseptic conditions, the intercostobrachial nerve was cut proximally and the median nerve was redirected (end-to-end anastomosed) to the distal radial nerve stump at the denervated triceps. The ulnar and radial nerves were cut distally and sutured beneath the normally innervated pectoralis muscle to prevent them from taking part in the targeted reinnervation nerve regeneration. After nerve redirection, the animals underwent an elbowlevel amputation. Following recovery, animals were caged individually with ad libitum access to food and water until their use in electrophysiological recording experiments.

Amputation without targeted reinnervation. Amputation-only animals (average weight $=378.3 \mathrm{~g}, \mathrm{SD}=12.9$ ) were anesthetized and prepared as described above. The median and ulnar nerves were sectioned at the elbow and the radial nerve was sectioned at the triceps. All of the nerves were sutured beneath the normally innervated pectoralis muscle, and we performed an elbow-level amputation. These steps served to silence the input to cortex from the forepaw and distal forearm and prevent any reinnervation at the distal residual limb arising from the sectioned nerves. The rats were closed and recovered as described above until recording experiments.

Electrophysiological recording. After a minimum of 13 weeks, all 18 animals (average weight $=496.6 \mathrm{~g}, \mathrm{SD}=23.5$ ) were anesthetized with an initial dose of $30 \%$ urethane $(1.5 \mathrm{~g} / \mathrm{kg})$ supplemented with doses of 22.5 $\mathrm{mg} / \mathrm{kg}$ ketamine hydrochloride and $3 \mathrm{mg} / \mathrm{kg}$ xylazine as needed to maintain a surgical plane of anesthesia. Body temperature was maintained with a water-circulating heating pad. A craniotomy was performed over the left hemisphere and the brain was covered with silicon oil. An enlarged photograph of the cortical surface was used to mark electrode penetrations.

Multiunit recordings were made $\sim 700 \mu \mathrm{m}$ deep to the pial surface in the primary somatosensory cortex using a tungsten microelectrode (1.0 $\mathrm{M} \Omega$ at $1 \mathrm{kHz}$, Microprobes). Electrode penetrations were placed randomly within each mediolateral and rostrocaudal search line. Multiunit activity was amplified and passed to an oscilloscope, a Cambridge Elec- 
tronic Design Power 1401 computer interface and an audio speaker. The receptive fields for each electrode penetration were determined by brushing the body surface with a handheld probe, and the borders were drawn on schematic diagrams of an amputated rat (nonresponsive sites were also noted).

Electrolytic lesions were placed in cortex and later used to align the electrode penetrations to the cortical histology. Following recording, the animals were killed with sodium pentobarbital $(100 \mathrm{mg} / \mathrm{kg}$ ) and perfused through the heart with $1 \times$ PBS, pH 7.4, followed by $4 \%$ paraformaldehyde (PFA) in PBS. The cerebral hemisphere was removed and fixed overnight in $4 \%$ PFA followed by cryoprotection in $30 \%$ sucrose in PBS for $24 \mathrm{~h}$. The cortex was flattened on a freezing microtome and sectioned $(60 \mu \mathrm{m})$ parallel to the pial surface. Sections were processed for cytochrome oxidase [adapted from (Wong-Riley, 1979)] and mounted on Adhesion Superfrost Plus microscope slides (Brain Research Laboratories). Sections showing barrel subfields were visualized using a $2 \times$ objective on an Eclipse 50i microscope and photomosaic images of S1 were generated using NIS-Elements BR software (Nikon Instruments). Resulting images were converted to grayscale and processed for contrast and brightness in Photoshop CS2 (Adobe). The electrode penetration map was transferred to the digital photograph of the cortex and overlaid and aligned with the cortical histology using Illustrator CS2 (Adobe). Outlines were drawn around the cytochrome oxidase-delineated barrel fields, and the electrode penetration points were related to their position with respect to the morphological boundaries of $S 1$.

Latency measurements. In five of the targeted reinnervation animals, the latency to cortex from an electrically evoked potential applied to the residual limb was measured at every electrode penetration made across the forelimb barrel subfield. A 22 gauge stainless steel tubing bipolar stimulating electrode (tip-to-tip distance $=3.5 \mathrm{~mm}$ ) was used to deliver an electrical current from a DS3 ConstantCurrent stimulator (Digitimer) to the center of the receptive field on the surface of the reinnervated residual limb skin at $1.5 \times$ threshold ( $18 \mathrm{~mA}$ ) for $50 \mu$ s with an interstimulus interval of $500 \mathrm{~ms}$. The latencies were measured using Spike 2 software starting at the onset of the stimulus artifact and averaged over 25 stimulus applications and mapped to their penetration location within S1 using Matlab (MathWorks).
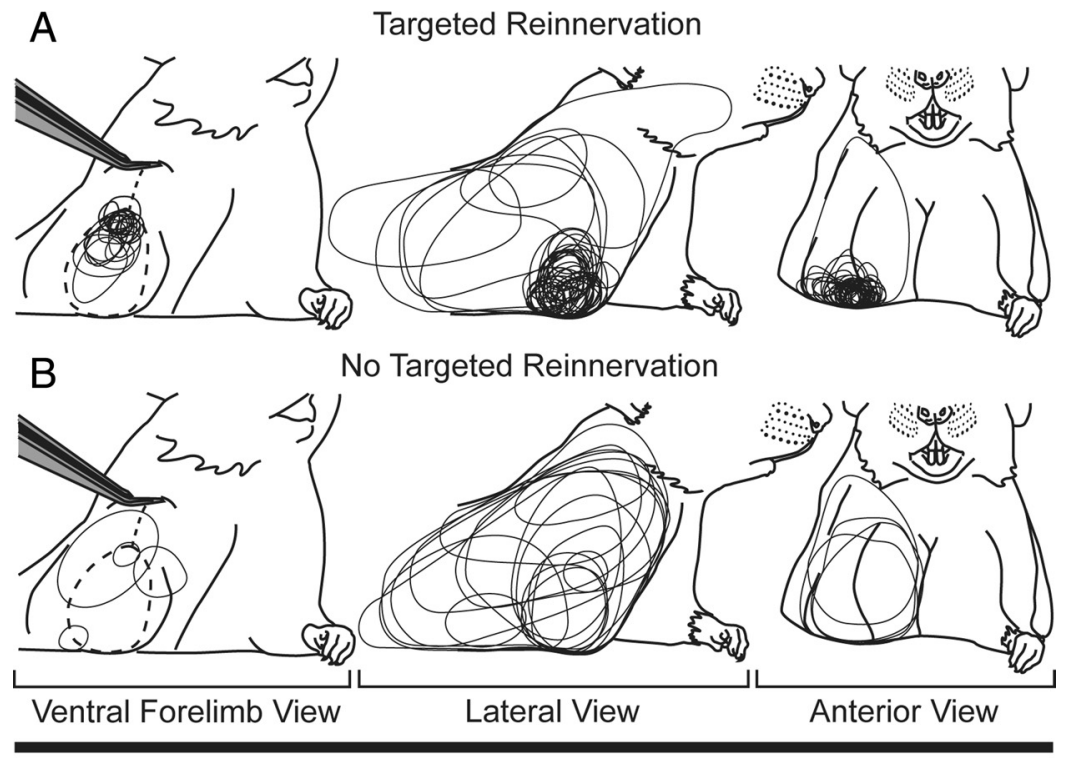

C

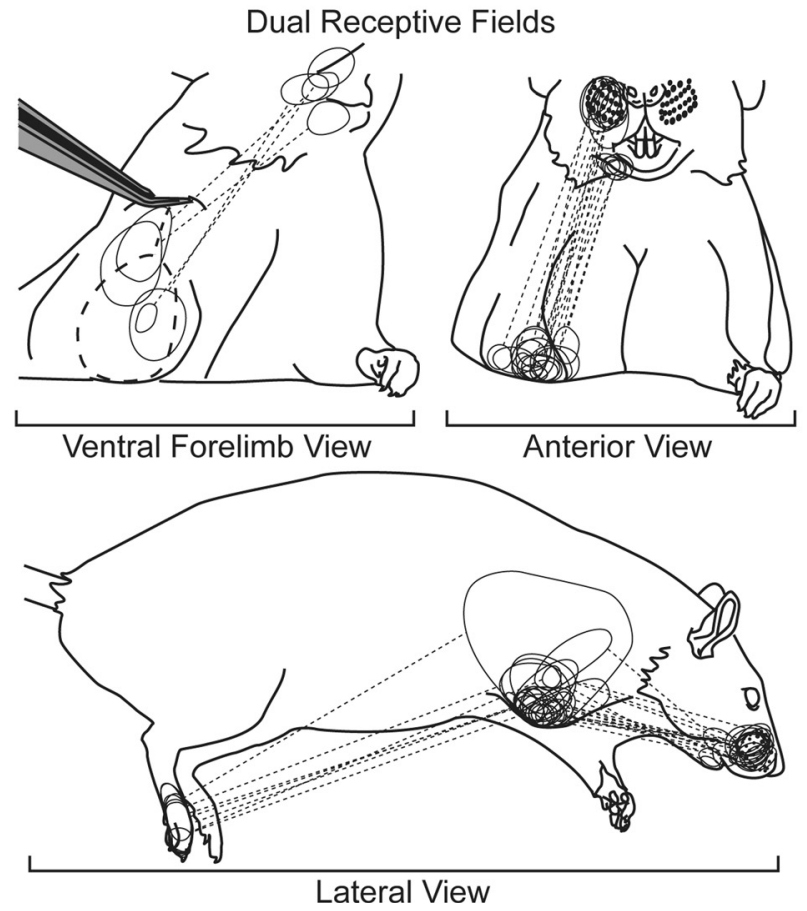

Figure 2. Morphology of the receptive fields on the residual limbs of the study animals (ventral, lateral, and anterior views). Tweezers are shown holding up residual limb to expose ventral surface (dotted line). $\boldsymbol{A}$, Composite receptive fields for three randomly chosen targeted reinnervation animals (106 penetrations). Receptive fields are small and are tightly clustered at the distal residual limb in the region denervated by section of the intercostobrachial nerve. These small receptive fields were almost exclusively recorded from within the forelimb barrel subfield. $\boldsymbol{B}$, Composite receptive fields for three randomly chosen nontargeted reinnervation control animals (25 penetrations). Receptive fields are large and distributed evenly across the shoulder and residual limb. C, Composite of dual receptive fields for all targeted reinnervation animals. Dotted lines connect the receptive field on the residual limb to the associated receptive field on the distant body surface. Receptive fields were found exclusively on either the hindpaw, chin/lower lip, or vibrissae.

\section{Results}

\section{Cortical mapping in adult male Sprague Dawley rats}

To investigate the effect of targeted reinnervation on S1 organization, we performed electrophysiological cortical recording in 18 adult Sprague Dawley rats. Of the 18 animals, 14 underwent amputation with targeted reinnervation and 4 underwent amputation-only control surgeries. We collected 1975 multiunit electrophysiological recording points across the 18 study animals. In the 14 targeted reinnervation animals, an average of 100.5 penetrations were collected per animal (range $=79-$ 118). In the 4 control animals, an average of 142.0 penetrations were collected per animal (range $=140-144$ ). In all animals, the highest density of points was collected across the forelimb barrel subfield, the region of the brain that processes touch input from the forepaw (point-to-point distance $=250-300 \mu \mathrm{m}$ ) with a lower density distributed across the surrounding extent of the S1 body representation (point-to-point distance $=500-550 \mu \mathrm{m}$ ). 
A

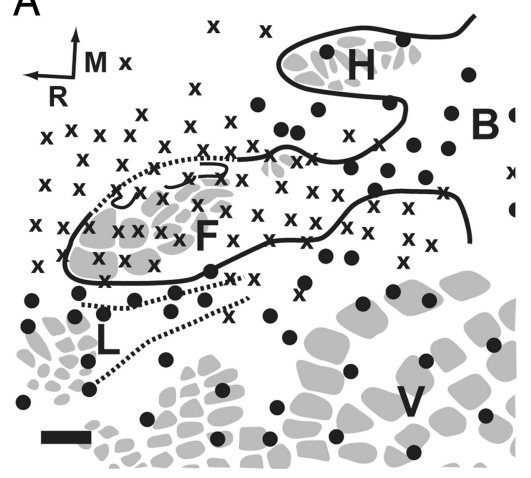

C

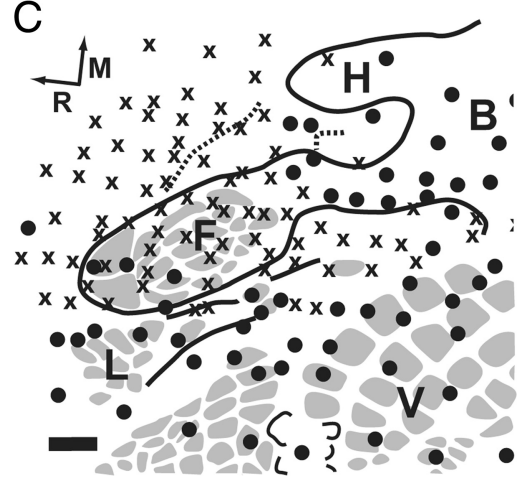

B

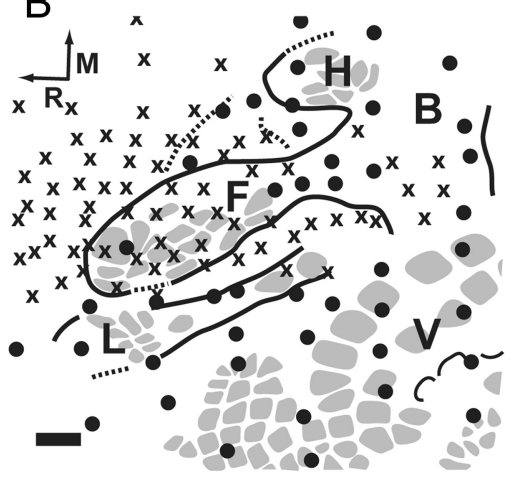

$\mathrm{D}$

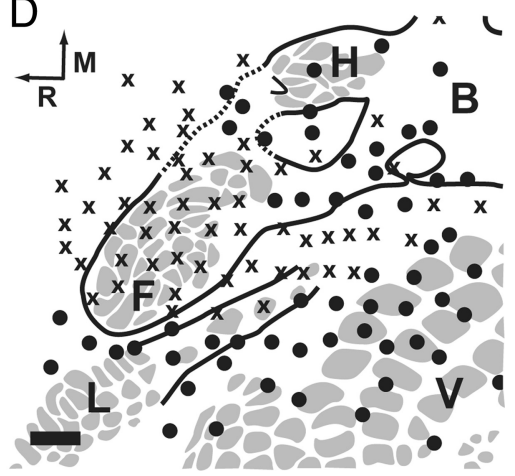

Figure 3. Results of microelectrode recordings in $S 1$ cortex of the left hemisphere of the four amputated control rats $(\boldsymbol{A}-\boldsymbol{D})$. See Figure $1 A$ for complete map of $S 1$ organization. Gray spots delineate barrel patterns within $S 1$. $\bullet$, Responsive site; $X$, unresponsive site; $F$, forepaw barrel subfield. Barrel patterns within the forelimb barrel subfield correspond to digits of the forepaw; $H$, hindpaw barrel subfield; B, body; V, vibrissae; L, chin/lower lip; R, rostral; M, medial. The forelimb barrel subfield was found to be largely silent in these animals. Cytochrome oxidase-delineated morphological boundaries appeared intact. Scale bars, $500 \mu \mathrm{m}$.

\section{Cortical maps: amputated animals with targeted reinnervation}

A group of 14 animals underwent an amputation with targeted reinnervation. Following recovery from nerve redirection and amputation, the targeted reinnervation animals underwent cortical recording procedures. The forelimb barrel subfields of the 14 targeted reinnervation animals were found to be mostly active (Fig. 1). In nine of the cases, although the forelimb barrel subfield was still largely active, there were some sites in the forelimb barrel subfield with no recorded activity (Fig. $1 B, C, G-J, L, N, O$ ). The receptive fields for recording sites in the forelimb barrel subfield were localized to the distal residual limb. They were characteristically small and overlapping (Fig. 2A). These small receptive fields were primarily isolated to the skin that was previously denervated by sectioning the intercostobrachial nerve. There were points with no activity in the shoulder representation between the active regions of the forelimb barrel subfield and the body in five animals (Fig. 1I,L-O); however, in all others, activity was contiguous from the forelimb barrel subfield to the body representation.

Eleven of the targeted reinnervation animals had electrode penetrations in the forelimb barrel subfield that were simultaneously responsive both to the distal residual limb and to other sites on the face and hindlimb (Fig. 2C). There was an average of 2.8 electrode penetrations (range 1-6) per animal showing dual receptive fields (Fig. 1, see points marked with $\boldsymbol{\bullet}$ ). Dual receptive fields were typically found near the boundaries of the forelimb barrel subfield at distances no further than $250 \mu \mathrm{m}$ from the histologically determined edge of the representation. Electrode penetrations recording activity from residual limb and hindfoot were found between the forelimb barrel subfield and the hind- limb barrel subfield. Electrode penetrations recording activity from either the vibrissae and residual limb or the lower lip and residual limb were clustered at the lateral edge of the forelimb barrel subfield near the lower lip. Many vibrissae and residual limb responses were found near the lateral/caudal edge of the forelimb barrel subfield between the forelimb barrel subfield and the vibrissae/buccal pad representations, while some showed recorded activity from the residual limb and vibrissae/buccal pad, but not from lower lip as might be expected.

\section{Cortical maps: amputated control} animals without targeted reinnervation A group of four control animals underwent an amputation only with no targeted reinnervation to provide comparison to the S1 cortex when amputated forelimb nerves were not allowed to reinnervate the residual limb skin. All of the cut nerves were sutured beneath the normally innervated pectoralis muscle, and an elbowlevel amputation was performed. This served to silence the input to cortex from the forepaw and forearm. Following postamputation recovery on the same time scale as the targeted reinnervation animals the control animals underwent cortical recording procedures. The forelimb barrel subfield in the four control animals was largely silenced. No activity was recorded at most electrode penetration sites within the boundaries of the forelimb barrel subfield the extent of which was histologically defined after the termination of the experiment with cytochrome oxidase (Fig. 3). Receptive fields on the shoulder and residual limb mapped to penetrations in the $\mathrm{S} 1$ shoulder region between the forelimb barrel subfield and the trunk representation. Similar patterns of S1 activity following amputation have been described by others (Welker, 1971; Hall and Lindholm, 1974; Dawson and Killackey, 1987). The receptive fields on the shoulder and residual limb were large and evenly distributed across the skin (Fig. $2 B$ ). The size and extent of the receptive fields on the residual limb and shoulder were similar to those observed on the rest of the body.

\section{Evoked response latencies in amputated animals with targeted reinnervation}

Latencies to cortex from electrically evoked somatosensory potentials on the reinnervated limb were used to examine connectivity from the afferents at the reinnervated target skin surface to the forelimb barrel subfield (Fig. 4). Average evoked response latency measurements (from stimulus artifact to cortical response) within the forelimb barrel subfield of the five animals ranged on an even continuum from $6.6 \mathrm{~ms}$ to $23.0 \mathrm{~ms}$ (Fig. 4A). The overall average of the latencies was $12.0 \mathrm{~ms}(\mathrm{SD}=3.8 \mathrm{~ms}$ ). Each animal showed a mix of both shorter and longer average latencies to cortex. However, animal TR-14 had $39.0 \%$ of penetrations with latencies under $9.0 \mathrm{~ms}$ and $55.0 \%$ of penetrations with latencies under $10.0 \mathrm{~ms}$ (Fig. 4C). Across all the animals, slightly over one-third of the latencies (36.4\%) were between 6.5 and $10.0 \mathrm{~ms}$, and one-third (33.3\%) were between 12.0 and 18.0 
ms. Of the remaining third, $19.7 \%$ fell between 10.0 and $12.0 \mathrm{~ms}$ and $10.6 \%$ were $>18.0 \mathrm{~ms}$. Two of the five animals had dual receptive fields, allowing recording of evoked response latencies for both the receptive field on the residual limb and the receptive field on the lower lip at a single cortical electrode penetration (Figs. $1 J, L, 4 C, F)$. In animal TR-11 at penetration 72 , the average latency for the residual limb was $12.7 \mathrm{~ms}$ and $9.2 \mathrm{~ms}$ for the lower lip. In animal TR-14 at penetrations 47 and 54, the average latencies were 8.7 $\mathrm{ms}$ and $11.3 \mathrm{~ms}$, respectively, for the residual limb and $4.7 \mathrm{~ms}$ and $5.2 \mathrm{~ms}$, respectively, for the lower lip.

\section{Discussion}

There was a difference in activity within the forelimb barrel subfield between the targeted reinnervation and control animals. The amputated control animals showed a near-complete silencing of the forelimb barrel subfield, and similarly to results reported by others, we found that the receptive fields for the few electrode penetrations that were active in the forelimb barrel subfield of the control animals were located primarily on the shoulder (Pearson et al., 1999). However, the extent of the activation of forelimb barrel subfield in our control animals is somewhat lower and may be related to the aggressive steps taken to remove possible input from the transected nerves. In contrast to controls, the targeted reinnervation animals showed extensive activity in the forelimb barrel subfield. The small receptive fields were tightly clustered on the distal residual limb of the targeted reinnervation animals and differed in proportion from the large and diffuse receptive fields observed on the residual limb of the control animals (Fig. 2A,B). The appearance of small receptive fields seemed similar to observations in nerve section studies in nonhuman primates (Merzenich et al., 1983). The redirection point of the distal median nerve was the only difference between the targeted reinnervation and control animals, suggesting that the reactivation of the forelimb barrel subfield in the targeted reinnervation animals occurred solely through the input of the median nerve. In these animals, the sensory input from the reinnervated target skin appeared to be processed within the cortical representation of the forepaw. In light of these results, it seems possible that when human targeted reinnervation amputees report feeling stimulation of their reinnervated skin on their missing hand, it is because the redirected afferent input is being processed within their central hand representation.

The extent of reactivation of forelimb barrel subfield cortex by median nerve redirection in the targeted reinnervation animals was marked. It has been demonstrated that restricted deafferentation of limb nerves in nonhuman primates allows remaining afferents to expand their cortical territory (Merzenich et al., 1983). Incomplete dorsal column section in nonhuman primates also produces similar results (Jain et al., 1997). Afferents from the
B

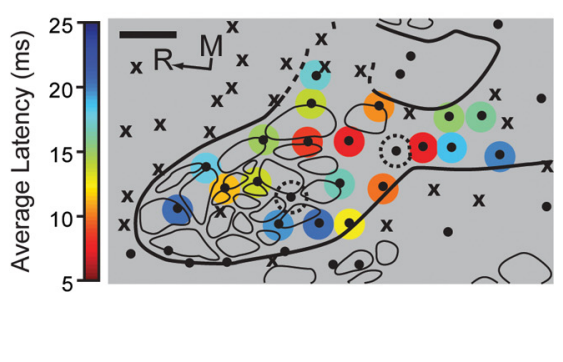

D

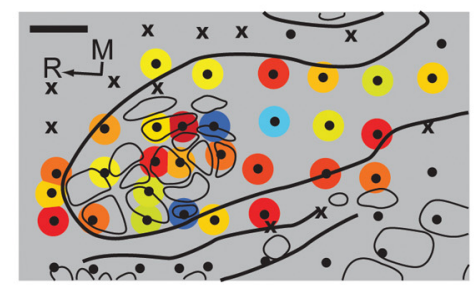

F

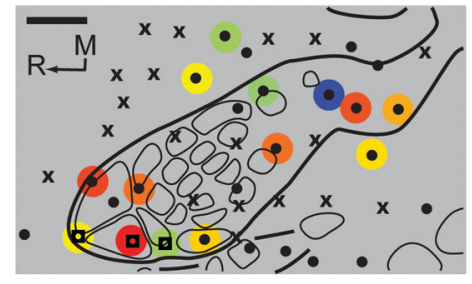

Figure 4. Latency measurements for electrically evoked somatosensory potentials from stimulus artifact to cortical response from the reinnervated target skin to the electrode penetration points within the forelimb barrel subfield of five targeted reinnera each penetration range from $6.6 \mathrm{~ms}$ to $23.0 \mathrm{~ms}$. Often, shorter latencies lie directly adjacent to longer latencies. In $\boldsymbol{F}$, no latencies were recorded at four points within the forelimb barrel subfield (see $\bullet$ ). Scale bars, $500 \mu \mathrm{m}$.

skin are linked directly to specific representations in S1 but also communicate more broadly through alternate connections to other areas within S1. This interconnectivity likely accounts for the representational expansion often seen following loss of afferent input (Garraghty et al., 1994; Lane et al., 1995; Schroeder et al., 1995; Hickmott and Merzenich, 1998; Li et al., 2002). Similar avenues of expansion may be involved with the reactivation of cortex seen in these targeted reinnervation animals. Interestingly, in five of the targeted reinnervation rats, there was a region of silent cortex between the active forelimb barrel subfield and the intact body representation, suggesting that there may be some limit to the expansion of activity from the reinnervated inputs (Fig. $1 I, L-O)$. In 11 animals, regions of cortex at the borders of the forelimb barrel subfield showed electrode penetrations that were responsive to both the distal residual limb and other regions of the body, such as vibrissae, lower lip, and hindfoot (Figs. $1 B$ $D, F-H, J-L, N, 2 C)$. It has been demonstrated that following nerve crush or transection, adjacent cortical areas will expand into denervated regions, and then as the injured input recovers the inputs share space and appear to compete until a normal somatotopic organization returns (Kis et al., 1999; Farnè et al., 2002). The presence of dual receptive fields in these animals sug- 
gests that similar mechanisms of recovery and reorganization may be at work with this approach as well.

To explore possible avenues of connectivity, we measured latency to cortex of electrically evoked potentials from the reinnervated skin of the residual limb. The length of the latency correlates with connectivity. Shorter latencies are likely reflective of more direct or dominant connections from the skin to cortex, while longer latencies may relate to polysynaptic or nondominant pathways (Ebner and Armstrong-James, 1990; Schroeder et al., 1995). In the adult rat, evoked response latencies from the intact forelimb have been shown to take between 6.5 and $10.3 \mathrm{~ms}$ to reach cortex, while postamputation polysynaptic alternative plasticity pathways within S1 take between 12.5 and $18.0 \mathrm{~ms}$ to reach their destination (Pearson et al., 1999). We found evoked potential latencies from the residual limb of the targeted reinnervation animals that were evenly distributed between $6.6 \mathrm{~ms}$ to $23.0 \mathrm{~ms}$ across all five animals (Fig. 4A). Within each animal, there were points with shorter latencies intermixed with points having longer latencies. Latencies at adjacent penetrations did not appear to change along a smooth continuum. For instance, penetrations with latencies at different ends of the time spectrum were often lying in close apposition (Fig. $4 B-F$ ). We suggest that the short latency penetrations may be reflective of more direct afferent connections to the residual limb through the redirected nerves, while the longer latencies may be associated with the expansion of cortical territory from the reconnected afferents through alternate multisynaptic pathways.

Many of the human targeted reinnervation patients report that they feel large areas of their hand when a single point on their reinnervated skin is touched (Kuiken et al., 2007a). The projected sensations that cover large areas of the hand and limb may result from limited input from the skin surface activating anatomical interconnections between pathways or representations within the CNS (Wall et al., 1986; Merzenich et al., 1990; Merzenich and Jenkins, 1993; Hansson and Brismar, 2003). It is also possible that the projected sensations stem from a concentration of reinnervating afferents in the target skin (Horch, 1979; Horch and Burgess, 1980; Kuiken et al., 1995; Manger et al., 1996). Previous work with these amputees provides evidence that the receptor density in the reinnervated skin is reflective of the available receptor density of the target skin and not the former density of receptors in the amputated hand (Marasco et al., 2009). This suggests that there are a reduced number of afferents communicating to cortex from the skin surface. Similarly, the latency results from this study provide evidence that there are areas of direct connection to the skin surface intermixed with areas of expanded representation through multisynaptic interconnections. These data offer the possibility that the perception of large areas of the missing hand felt by these amputees may be a product of the activation of central anatomical interconnections.

It has been suggested that increasing the cortical territory devoted to a set of inputs may amplify their significance (Kaas, 1991; Jain et al., 1997). Increasing representational area appears to lead to a recruitment of processing resources (Godde et al., 1996; Dinse et al., 1997). For instance, providing more cortical territory to repaired hand nerves by anesthetizing the skin of the forearm improves sensory function (Rosén et al., 2006). An amplification of effectiveness from an expanded cortical representation may help to explain the increased tactile acuity of the reinnervated skin that has been observed in targeted reinnervation patients. These patients can localize point stimuli more effectively on their reinnervated skin than on their contralateral normal skin, even though the receptor density appears to be sim- ilar between the two skin surfaces (Marasco et al., 2009). These subjects also appear to be compensating for a disruption in the somatotopy of the reinnervated skin surface through central processing mechanisms (Marasco et al., 2009). The expansion of cortical territory seen in this study may provide a mechanism for this observed amplification of the functionality of the reinnervated target skin.

There is a strong desire on the part of upper limb amputees to have prosthetic devices that provide sensory feedback (Atkins et al., 1996; Biddiss et al., 2007), yet current prostheses lack this feature (Scott, 1990). Interfacing prosthetic touch feedback through physiologically relevant afferent channels may offer the opportunity to give the amputees the sense that their prosthetic device is connected to them more like it was their own hand. This may help improve function by providing touch feedback and helping to more effectively integrate the prosthetic device into the amputee's self image (Van Dorsten, 2004; Dhillon and Horch, 2005; Murray, 2008; Rybarczyk and Behel, 2008). The results of this study provide insight into how targeted reinnervation may be providing sensation that is perceived as being from the missing hand. These results also lend support to the idea that the reinnervated target skin could possibly serve as a direct line of communication from a prosthetic device to the hand and limb sensory processing regions of the brain.

\section{References}

Atkins D, Heard D, Donovan W (1996) Epidemiologic overview of individuals with upper-limb loss and their reported research priorities. J Prosthet Orthot 8:2-11

Biddiss E, Beaton D, Chau T (2007) Consumer design priorities for upper limb prosthetics. Disabil Rehabil Assist Technol 2:346-357.

Borsook D, Becerra L, Fishman S, Edwards A, Jennings CL, Stojanovic M, Papinicolas L, Ramachandran VS, Gonzalez RG, Breiter H (1998) Acute plasticity in the human somatosensory cortex following amputation. Neuroreport 9:1013-1017.

Cenci MA, Whishaw IQ, Schallert T (2002) Animal models of neurological deficits: how relevant is the rat? Nat Rev Neurosci 3:574-579.

Dawson DR, Killackey HP (1987) The organization and mutability of the forepaw and hindpaw representations in the somatosensory cortex of the neonatal rat. J Comp Neurol 256:246-256.

Dhillon GS, Horch KW (2005) Direct neural sensory feedback and control of a prosthetic arm. IEEE Trans Neural Syst Rehabil Eng 13:468-472.

Dinse HR, Godde B, Hilger T, Haupt SS, Spengler F, Zepka R (1997) Shortterm functional plasticity of cortical and thalamic sensory representations and its implication for information processing. Adv Neurol 73:159-178

Ebner FF, Armstrong-James MA (1990) Intracortical processes regulating the integration of sensory information. Prog Brain Res 86:129-141.

Farnè A, Roy AC, Giraux P, Dubernard JM, Sirigu A (2002) Face or hand, not both: perceptual correlates of reafferentation in a former amputee. Curr Biol 12:1342-1346.

Flor H, Elbert T, Mühlnickel W, Pantev C, Wienbruch C, Taub E (1998) Cortical reorganization and phantom phenomena in congenital and traumatic upper-extremity amputees. Exp Brain Res 119:205-212.

Flor H, Mühlnickel W, Karl A, Denke C, Grüsser S, Kurth R, Taub E (2000) A neural substrate for nonpainful phantom limb phenomena. Neuroreport 11:1407-1411.

Garraghty PE, Hanes DP, Florence SL, Kaas JH (1994) Pattern of peripheral deafferentation predicts reorganizational limits in adult primate somatosensory cortex. Somatosens Mot Res 11:109-117.

Godde B, Spengler F, Dinse HR (1996) Associative pairing of tactile stimulation induces somatosensory cortical reorganization in rats and humans. Neuroreport 8:281-285.

Hall RD, Lindholm EP (1974) Organization of motor and somatosensory neocortex in the albino rat. Brain Res 66:23-38.

Hansson T, Brismar T (2003) Loss of sensory discrimination after median nerve injury and activation in the primary somatosensory cortex on functional magnetic resonance imaging. J Neurosurg 99:100-105.

Hickmott PW, Merzenich MM (1998) Single-cell correlates of a representational boundary in rat somatosensory cortex. J Neurosci 18:4403-4416. 
Hijjawi JB, Kuiken TA, Lipschutz RD, Miller LA, Stubblefield KA, Dumanian GA (2006) Improved myoelectric prosthesis control accomplished using multiple nerve transfers. Plast Reconstr Surg 118:1573-1578.

Horch K (1979) Guidance of regrowing sensory axons after cutaneous nerve lesions in the cat. J Neurophysiol 42:1437-1449.

Horch KW, Burgess PR (1980) Functional specificity and somatotopic organization. In: Nerve repair and regeneration: its clinical and experimental basis (Jewett DL, McCarroll HR, eds), pp 105-114. St. Louis: Mosby.

Iwaniuk AN, Whishaw IQ (2000) On the origin of skilled forelimb movements. Trends Neurosci 23:372-376.

Jain N, Catania KC, Kaas JH (1997) Deactivation and reactivation of somatosensory cortex after dorsal spinal cord injury. Nature 386:495-498.

Kaas JH (1991) Plasticity of sensory and motor maps in adult mammals. Annu Rev Neurosci 14:137-167.

Kis Z, Farkas T, Rábl K, Kis E, Kóródi K, Simon L, Marusin I, Rojik I, Toldi J (1999) Comparative study of the neuronal plasticity along the neuraxis of the vibrissal sensory system of adult rat following unilateral infraorbital nerve damage and subsequent regeneration. Exp Brain Res 126:259-269.

Kuiken TA, Childress DS, Rymer WZ (1995) The hyper-reinnervation of rat skeletal muscle. Brain Res 676:113-123.

Kuiken TA, Dumanian GA, Lipschutz RD, Miller LA, Stubblefield KA (2004) The use of targeted muscle reinnervation for improved myoelectric prosthesis control in a bilateral shoulder disarticulation amputee. Prosthet Orthot Int 28:245-253.

Kuiken TA, Marasco PD, Lock BA, Harden RN, Dewald JP (2007a) Redirection of cutaneous sensation from the hand to the chest skin of human amputees with targeted reinnervation. Proc Natl Acad Sci USA 104:20061-20066.

Kuiken TA, Miller LA, Lipschutz RD, Lock BA, Stubblefield K, Marasco PD, Zhou P, Dumanian GA (2007b) Targeted reinnervation for enhanced prosthetic arm function in a woman with a proximal amputation: a case study. Lancet 369:371-380.

Lane RD, Bennett-Clarke CA, Chiaia NL, Killackey HP, Rhoades RW (1995) Lesion-induced reorganization in the brainstem is not completely expressed in somatosensory cortex. Proc Natl Acad Sci U S A 92:42644268.

Li CX, Callaway JC, Waters RS (2002) Removal of GABAergic inhibition alters subthreshold input in neurons in forepaw barrel subfield (FBS) in rat first somatosensory cortex (SI) after digit stimulation. Exp Brain Res 145:411-428.

Manger PR, Woods TM, Jones EG (1996) Plasticity of the somatosensory cortical map in macaque monkeys after chronic partial amputation of a digit. Proc Biol Sci 263:933-939.

Marasco PD, Schultz AE, Kuiken TA (2009) Sensory capacity of reinnervated skin after redirection of amputated upper limb nerves to the chest. Brain 132:1441-1448

Merzenich MM, Jenkins WM (1993) Reorganization of cortical representations of the hand following alterations of skin inputs induced by nerve injury, skin island transfers, and experience. J Hand Ther 6:89-104.

Merzenich MM, Kaas JH, Wall J, Nelson RJ, Sur M, Felleman D (1983) Topographic reorganization of somatosensory cortical areas $3 \mathrm{~b}$ and 1 in adult monkeys following restricted deafferentation. Neuroscience $8: 33-55$.

Merzenich MM, Recanzone GH, Jenkins WM, Nudo RJ (1990) How the brain functionally rewires itself. In: Natural and artificial parallel computation (Arbib M, Robinson JA, eds), pp 177-210. Cambridge: MIT Press.

Murray C (2008) Embodiment and prosthetics. In: Psychoprosthetics
(Gallagher P, Desmond D, MacLachlan M, eds), pp 119-129. London: Springer.

Paul RL, Goodman H, Merzenich M (1972) Alterations in mechanoreceptor input to Brodmann's areas 1 and 3 of the postcentral hand area of Macaca mulatta after nerve section and regeneration. Brain Res 39:1-19.

Pearson PP, Li CX, Waters RS (1999) Effects of large-scale limb deafferentation on the morphological and physiological organization of the forepaw barrel subfield (FBS) in somatosensory cortex (SI) in adult and neonatal rats. Exp Brain Res 128:315-331.

Ramachandran VS, Stewart M, Rogers-Ramachandran DC (1992a) Perceptual correlates of massive cortical reorganization. Neuroreport 3:583586.

Ramachandran VS, Rogers-Ramachandran D, Stewart M (1992b) Perceptual correlates of massive cortical reorganization. Science 258:1159-1160.

Robinson D (1968) The electrical properties of metal microelectrodes. Proc IEEE 56:1065-1071.

Rosén B, Björkman A, Lundborg G (2006) Improved sensory relearning after nerve repair induced by selective temporary anaesthesia-a new concept in hand rehabilitation. J Hand Surg Br 31:126-132.

Rybarczyk B, Behel J (2008) Limb loss and body image. In: Psychoprosthetics (Gallager P, Desmond D, MacLachlan M, eds), pp 23-31. London: Springer.

Schroeder CE, Seto S, Arezzo JC, Garraghty PE (1995) Electrophysiological evidence for overlapping dominant and latent inputs to somatosensory cortex in squirrel monkeys. J Neurophysiol 74:722-732.

Schultz AE, Marasco PD, Kuiken TA (2009) Vibrotactile detection thresholds for chest skin of amputees following targeted reinnervation surgery. Brain Res 1251:121-129.

Scott RN (1990) Feedback in myoelectric prostheses. Clin Orthop Relat Res 256:58-63.

Sensinger JW, Schultz AE, Kuiken TA (2009) Examination of force discrimination in human upper limb amputees with reinnervated limb sensation following peripheral nerve transfer. IEEE Trans Neural Syst Rehabil Eng 17:438-444.

Van Dorsten B (2004) Integrating psychological and medical care: practice recommendations for amputation. In: Functional restoration of adults and children with upper extremity amputation, Ed 1 (Meier R, Atkins D, eds), pp 73-88. New York: Demos Medical.

Wall JT, Cusick CG (1984) Cutaneous responsiveness in primary somatosensory (S-I) hindpaw cortex before and after partial hindpaw deafferentation in adult rats. J Neurosci 4:1499-1515.

Wall JT, Kaas JH, Sur M, Nelson RJ, Felleman DJ, Merzenich MM (1986) Functional reorganization in somatosensory cortical areas $3 \mathrm{~b}$ and 1 of adult monkeys after median nerve repair: possible relationships to sensory recovery in humans. J Neurosci 6:218-233.

Waters RS, Li CX, McCandlish CA (1995) Relationship between the organization of the forepaw barrel subfield and the representation of the forepaw in layer IV of rat somatosensory cortex. Exp Brain Res 103:183-197.

Welker C (1971) Microelectrode delineation of fine grain somatotopic organization of (SmI) cerebral neocortex in albino rat. Brain Res 26:259275.

Wong-Riley M (1979) Changes in the visual system of monocularly sutured or enucleated cats demonstrable with cytochrome oxidase histochemistry. Brain Res 171:11-28.

Zhou P, Lowery MM, Englehart KB, Huang H, Li G, Hargrove L, Dewald JP, Kuiken TA (2007) Decoding a new neural machine interface for control of artificial limbs. J Neurophysiol 98:2974-2982. 